\title{
Endoscopic full-thickness resection with defect closure using an over-the-scope clip for gastric subepithelial tumors originating from the muscularis propria
}

\author{
Jintao Guo $\cdot$ Zhijun Liu $\cdot$ Siyu Sun $\cdot$ \\ Xiang Liu $\cdot$ Sheng Wang $\cdot$ Nan Ge \\ Guoxin Wang $\cdot$ Yafei Qi
}

Received: 14 September 2014/Accepted: 12 January 2015/Published online: 21 February 2015

(C) The Author(s) 2015. This article is published with open access at Springerlink.com

\begin{abstract}
Background Endoscopic full-thickness resection (EFTR) is a mini-invasive technique for gastric subepithelial tumors originating from the muscularis propria, which enables a full-thickness resection of tumors and can provide a complete basis for pathological diagnosis. Gastric fistula closure after EFTR is a challenge for endoscopists. In this study, we introduced EFTR with fistula closure using the over-thescope clip (OTSC) system for gastric subepithelial tumors originating from the muscularis propria.

Objectives To evaluate the feasibility and safety of fistula closure with OTSC by a retrospective analysis on the cases of EFTR with defect closure using OTSC for gastric subepithelial tumors originating from the muscularis propria in our hospital. Methods The patients were selected who underwent EFTR for gastric subepithelial tumors originating from the muscularis propria (tumor diameter $\leq 2 \mathrm{~cm}$ ) in our hospital from October 2013 to March 2014. After a full-thickness
\end{abstract}

Electronic supplementary material The online version of this article (doi:10.1007/s00464-015-4076-2) contains supplementary material, which is available to authorized users.

J. Guo $\cdot$ S. Sun $(\varangle) \cdot$ X. Liu $\cdot$ S. Wang $\cdot$ N. Ge $\cdot$ G. Wang

Endoscopic Center, Shengjing Hospital of China Medical

University, No. 36 Sanhao Street,

Shenyang 110004, Liaoning Province, China

e-mail: sunsy@sj-hospital.org; sun-siyu@ 163.com

J. Guo

e-mail: guojt@sj-hospital.org

X. Liu

e-mail: liuxiangcmu@163.com

S. Wang

e-mail: wangshengcmu@163.com

N. Ge

e-mail: gen@sj-hospital.org resection of tumors, the bilateral gastric mucous membranes of defect were clamped using twin graspers and then drawn into the transparent cap of OTSC, and the OTSC was released to close the defect after full suctioning. The success rate of defect closure with OTSC was observed, and the endoscopic follow-up was performed at 1 week, 1 and 6 months after operation to check OTSC closure.

Results Totally 23 patients were included into the study. The full-thickness resection rate of gastric tumors in the muscularis propria was $100 \%(23 / 23)$, the success rate of defect closure was $100 \%$, and the average time of defect closure was $4.9 \mathrm{~min}$ (range 2-12 $\mathrm{min}$ ). All patients experienced no postoperative complications such as bleeding and perforation. The postoperative follow-up time was 1-6 months (mean 3 months), and no OTSC detachment was found.

Conclusions OTSC can be used to perform EFTR with defect closure for gastric tumors in the muscularis propria (tumor diameter $\leq 2 \mathrm{~cm}$ ). It is simple, convenient, safe and effective.

Keywords OTSC $\cdot$ Stomach $\cdot$ Submucosal tumor . Muscularis propria $\cdot$ Full-thickness resection

G. Wang

e-mail: wangguoxincmu@163.com

Z. Liu

Ultrasound Department, Shengjing Hospital of China Medical University, No. 36 Sanhao Street,

Shenyang 110004, Liaoning Province, China

e-mail: liuzj1@sj-hospital.org

Y. Qi

Pathological Department, Shengjing Hospital of China Medical

University, No. 36 Sanhao Street,

Shenyang 110004, Liaoning Province, China

e-mail: liuwencmu@163.com 
A gastrointestinal stromal tumor originating from the muscularis propria can be malignant; therefore, a reliable, full-thickness tumor resection is required. Considering the above, surgical and laparoscopic procedures are currently important techniques to treat larger gastric stromal tumors. For small, asymptomatic gastric tumors in the muscularis propria, however, it remains controversial whether to choose resection or regular follow-up [1]. In addition, this difficult decision is usually made by the patient.

In recent years, endoscopic dissection or an enucleation technique has been used for the resection of gastrointestinal tumors in the muscularis propria; however, gross resection ( $\mathrm{R} 1$ resection) is often successfully performed under endoscopy in most cases; complete resection (R0 resection) can be achieved only in a few cases. Endoscopic full-thickness tumor resection (and obtaining peri-tumor normal gastric tissues) could be a more minimally invasive choice for patients, provided that it could achieve the same therapeutic effect as a laparoscopic procedure for submucosal tumors. However, closing gastric defects quickly and reliably after full-thickness tumor resection is a challenge for endoscopists. In the present study, we proposed a new method to close gastric defects after a full-thickness gastric resection, i.e., defect closure with the over-the-scope clip (OTSC).

The OTSC closure system (Ovesco Endoscopy GmbH, Tuebingen, Germany) has been successfully applied to the treatment of gastrointestinal bleeding, fistulas and perforations [2]. For resection of gastrointestinal submucosal tumors not originating from the muscularis propria and wound closure, the feasibility of the OTSC closure system has been proven [3]. However, the application of the OTSC closure system in endoscopic full-thickness resection (EFTR) with gastric fistula closure for gastric tumors in the muscularis propria has been rarely described. Previous studies have reported that the metal clip was often used to close gastric fistula after EFTR [4].

In order to evaluate the safety and efficacy EFTR, we retrospectively analyzed the cases that underwent the procedure with defect closure using OTSC for gastric tumors in the muscularis propria.

\section{Patients and methods}

Patient data

We retrospectively analyzed the patients who underwent EFTR for gastric tumors in the muscularis propria (tumor diameter $\leq 2 \mathrm{~cm}$ ) in our hospital from October 2013 through March 2014. Before EFTR, all patients received computed tomography (CT) and endoscopic ultrasound (EUS) examinations to determine the originating layer of the gastric tumors, their relationship with adjacent great vessels of the stomach and the presence/absence of peripheral lymph node enlargement; thus, we excluded all malignant diseases. If a tumor originated from the muscularis propria (tumor diameter $\leq 2 \mathrm{~cm}$ ) and there was no peripheral lymph node enlargement and metastasis, EFTR was performed. All patients were hospitalized for treatment.

This study was approved by the Institutional Review Board and Ethics Committee of China Medical University. All patients voluntarily chose their therapeutic course and provided written informed consent for their participation in this study. The operator performing the EFTR procedure in this study was familiar with both endoscopic submucosal dissection and EFTR techniques.

Endoscopic equipment and accessories

A standard single-channel gastroscope (EPK-i, Pentax) was used throughout the endoscopic procedure; a linear array ultrasonic endoscope (EG3830UR; Pentax Precision Instrument, Orangeburg, New York) was used for the evaluation of tumor size, echo characteristics and the originating layer. A triangle-tipped knife and insulated-tip knife (both from Olympus Corporation, Tokyo, Japan) was used for the dissection and resection of tumors, a hot biopsy forceps (FD410LR) was used for gastric wall hemostasis, twin graspers (Ovesco Endoscopy GmbH, Tuebingen, Germany) were used for clamping the two sides of the gastric defect, and metal clips (Olympus Corporation, Tokyo, Japan) and the OTSC system (Ovesco Endoscopy GmbH, Tuebingen, Germany) were used for defect closure.

\section{Procedure}

\section{EFTR}

The peri-tumor gastric tissues were incised with a triangletipped knife, and then, the tumor and peri-tumor gastric tissues were gradually resected in full thickness using an insulated-tip knife (with the aim of protecting adjacent organs beneath the gastric wall) (Fig. 1a-e). An iatrogenic gastric perforation was created (Fig. 1f). During the resection, hemostasis was obtained with electric cautery.

\section{Wound closure with the OTSC system}

Gastric tissues adjacent to the iatrogenic perforation were clamped with twin graspers and then drawn into the transparent cap of the OTSC device; then, they were fully aspirated into the transparent cap, and the OTSC closure system was released to close the defect (Fig. 1g-i). If defect closure was incomplete, metal clips were used to close the remaining portions. After the gastric defect was completely closed, gastric gas was fully aspirated and the gastroscope was withdrawn. 

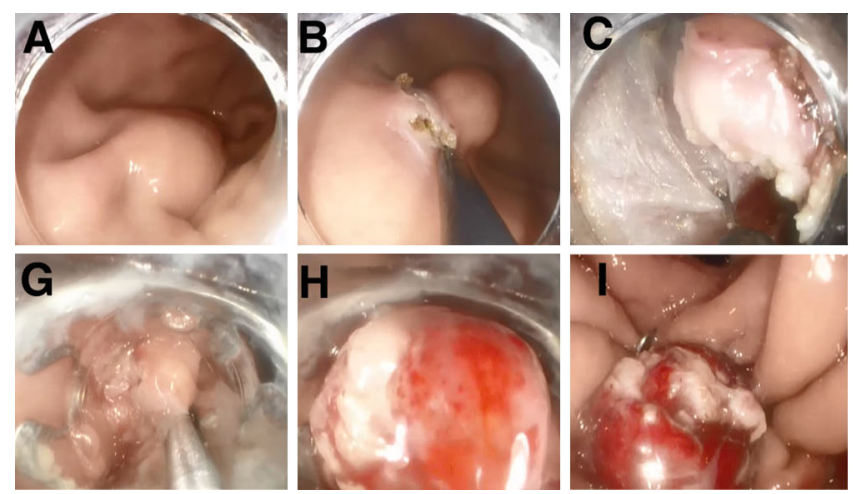

Fig. 1 A A protruding tumor with a smooth surface in the gastric fundus B Peri-tumor gastric mucosa was incised $\mathbf{C}$ Peri-tumor gastric submucosa was dissected D, E Full-thickness resection was performed around the tumor $\mathbf{F}$ Gastric defect formed after full-thickness tumor resection. The gastric walls at both margins of the defect were clamped with twin graspers $(\mathbf{G})$ and drawn into the transparent cap of

All EFTR and OTSC closure system operations were performed by one experienced endoscopist. Carbon dioxide was injected throughout the procedure. A 20-ml syringe was used to aspirate free gas from the abdomen during or after the procedure. A gastric decompression tube, which could also observe postoperative bleeding, was indwelled into the stomach. The postoperative treatment included $24 \mathrm{~h}$ of fasting (both food and water) as well as routine administration of proton pump inhibitors (PPIs) and antibiotics. All patients were closely observed. If significant hemorrhage occurred, hemostasis was obtained endoscopically or surgically. If peritonitis symptoms were observed, the indwelling time of the gastric decompression tube and the duration of antibiotic administration were usually increased. If conservative treatment was unsuccessful, surgical treatment was performed. On postoperative day 2, the patients without postoperative bleeding and peritonitis received a liquid diet and oral PPIs. If the patients did not experience discomfort and had normal laboratory studies after resuming their diet, they continued taking oral PPIs for 1 month after discharge.

At 1 week, 1 and 6 months postoperatively, endoscopic follow-up was performed to assess the wound and defect closure (Fig. 1k and 1).

\section{Statistical analysis}

Statistical analysis was performed with the SPSS software package version 19.0 (SPSS Inc., Chicago, IL, USA).

\section{Results}

This study comprised 23 patients aged $54.8 \pm 9.7$ (range $32-70)$ years, including 13 males $(57 \%)$ and 10 females
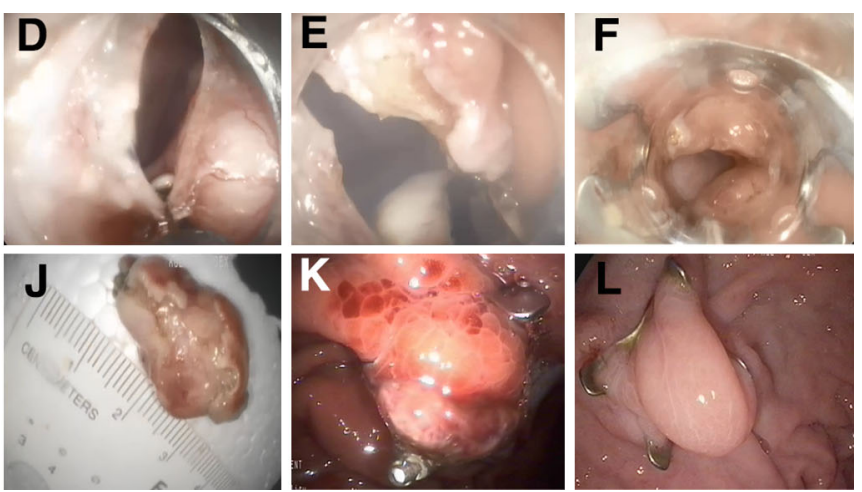

the OTSC for full suction (H) I OTSC clamping device was released to approximate the gastric walls at the defect $\mathbf{J}$ The full-thickness structure of the peri-tumor gastric wall is observed $\mathbf{K}$ Gastroscopic reexamination 1 week postoperatively revealed edema of the gastric wall $\mathbf{L}$ Gastroscopic reexamination 6 months postoperatively revealed a nearly normal gastric wall

(44\%). The mean tumor diameter was $12.1 \pm 4.7$ (range 6-20) $\mathrm{mm}$. The tumor was located in the gastric antrum in three cases, in the gastric body in nine cases, and in the gastric fundus in 11 cases. The full-thickness resection rate of gastric tumors in the muscularis propria was 23/23 (100\%), and the EFTR time was $40.5 \pm 25.8$ (range 16-104) $\mathrm{min}$. The rate of no residual tumor at the cutting edge was 23/23 (100\%) as documented by pathological diagnosis. Nineteen cases $(19 / 23 ; 83 \%)$ were stromal tumors, in which one case $(1 / 19 ; 5 \%)$ was at high risk and the remaining cases $(18 / 19 ; 95 \%)$ were at very low risk; four cases $(4 / 23 ; 17 \%)$ were leiomyomas. The success rate of defect closure was $100 \%$. In 23 patients, complete defect closure was achieved with only one OTSC. The defect closure time was $4.9 \pm 2.2$ (range 2-12) min. The incidence of fever $\left(T>37^{\circ} \mathrm{C}\right)$ was $3 / 23(13 \%)$ on the day of operation, $1 / 23$ ( $4 \%$ ), on postoperative day 1 , and $0 / 23(0 \%)$ on postoperative day 2 . Two patients $(9 \%)$ experienced localized peritonitis postoperatively and improved following conservative treatment. None of the patients developed postoperative bleeding or perforation. The mean postoperative hospital stay was 3 (range 2-5) days. The postoperative follow-up time was 3 (range 1-6) months, and no patient had OTSC detachment during follow-up. Patient demographics, lesion features, pathological diagnosis and clinical outcomes are summarized in Table 1 .

\section{Discussion}

There are various types of gastric tumors originating from the muscularis propria, among which a potential malignant tumor, i.e., gastrointestinal stromal tumor, is the most 
Table 1 Demographic and clinicopathological characteristics of the study patients $(N=23)$

\begin{tabular}{|c|c|c|c|c|c|c|}
\hline $\begin{array}{l}\text { Case } \\
\text { no. }\end{array}$ & $\begin{array}{l}\text { Gender/ } \\
\text { age (years) }\end{array}$ & Location & $\begin{array}{l}\text { Tumor size } \\
\text { (diameter, } \mathrm{mm} \text { ) }\end{array}$ & $\begin{array}{l}\text { Defect closure } \\
\text { time (min) }\end{array}$ & $\begin{array}{l}\text { Operation } \\
\text { time (min) }\end{array}$ & $\begin{array}{l}\text { Pathological } \\
\text { diagnosis }\end{array}$ \\
\hline 1 & $\mathrm{M} / 60$ & Antrum & 20 & 12 & 104 & GIST \\
\hline 2 & $\mathrm{~F} / 46$ & Body & 20 & 7 & 87 & GIST \\
\hline 3 & $F / 32$ & Body & 17 & 6 & 63 & GIST \\
\hline 4 & $\mathrm{M} / 38$ & Fundus & 6 & 6 & 39 & Leiomyoma \\
\hline 5 & $\mathrm{~F} / 42$ & Fundus & 12 & 8 & 42 & GIST \\
\hline 6 & $\mathrm{M} / 69$ & Body & 11 & 3 & 35 & GIST \\
\hline 7 & $\mathrm{M} / 63$ & Body & 14 & 4 & 31 & GIST \\
\hline 8 & M/66 & Fundus & 8 & 4 & 19 & Leiomyoma \\
\hline 9 & $\mathrm{M} / 47$ & Fundus & 8 & 6 & 26 & GIST \\
\hline 10 & $\mathrm{~F} / 52$ & Antrum & 16 & 3 & 103 & GIST \\
\hline 11 & $\mathrm{M} / 54$ & Body & 20 & 4 & 47 & GIST \\
\hline 12 & $\mathrm{~F} / 57$ & Body & 6 & 4 & 17 & GIST \\
\hline 13 & $\mathrm{M} / 59$ & Body & 13 & 7 & 27 & GIST \\
\hline 14 & $\mathrm{M} / 65$ & Body & 7 & 3 & 22 & GIST \\
\hline 15 & $\mathrm{M} / 62$ & Fundus & 6 & 3 & 31 & GIST \\
\hline 16 & $\mathrm{M} / 50$ & Body & 12 & 4 & 29 & GIST \\
\hline 17 & $\mathrm{~F} / 54$ & Fundus & 8 & 2 & 16 & GIST \\
\hline 18 & $\mathrm{M} / 70$ & Antrum & 14 & 6 & 36 & Leiomyoma \\
\hline 19 & $\mathrm{~F} / 49$ & Fundus & 10 & 5 & 25 & GIST \\
\hline 20 & $\mathrm{~F} / 56$ & Fundus & 14 & 5 & 31 & GIST \\
\hline 21 & $\mathrm{~F} / 51$ & Fundus & 7 & 4 & 18 & Leiomyoma \\
\hline 22 & $\mathrm{M} / 60$ & Fundus & 17 & 3 & 56 & GIST \\
\hline 23 & $\mathrm{~F} / 58$ & Fundus & 12 & 3 & 28 & GIST \\
\hline
\end{tabular}

common type [5, 6]. Resection is recommended for gastric tumors in the muscularis propria that are large and/or symptomatic; however, either long-term endoscopic follow-up or resection may be selected for those that are small and asymptomatic [1]. A disadvantage of long-term endoscopic follow-up is its burden on the patient. Currently, the imaging procedures (CT and EUS) cannot accurately determine the pathological diagnosis of submucosal tumors, and it is difficult to biopsy small tumors under EUS [7, 8]. The patients often express concern regarding tumor growth; thus, they are impacted with a psychological burden. Moreover, the tumors suspected to be gastric stromal tumors have the risk of growth and malignant transformation. Based on the above reasons, if a minimally invasive technique is available to resect even small gastric tumors in the muscularis propria, most patients prefer resection over long-term endoscopic followup.

Studies have demonstrated that endoscopic submucosal dissection (ESD) and endoscopic submucosal enucleation (ESE) are feasible for the resection of gastric tumors in the muscularis propria $[9,10]$. However, it is difficult to achieve R0 resection (resection of tumor and peri-tumor normal gastric tissues) by endoscopic dissection; thus, the risk of recurrence exists. Of course, laparoscopic surgery can attain R0 resection; however, it is inferior for the detection of small tumors and is also more invasive than endoscopic resection. In recent years, EFTR has been used for full-thickness resection of gastric tumors in the muscularis propria [11-14]. EFTR enables full-thickness resection of tumors; thus, it provides for attainment of a complete tumor specimen for pathologic study. EFTR facilitates the determination of whether residual tumor is present at the resection margins and also determines whether there is a need for subsequent treatment; thus, it reduces the possibility of residual tumor and recurrence. Shi et al. [11] successfully performed 20 cases of EFTR for gastric tumors in the muscularis propria; they reported a full-thickness resection rate of $100 \%$ and diameters of resected tumors of $1.47 \pm 0.72 \mathrm{~cm}$. Zhou et al. [12] resected gastric tumors originating from the muscularis propria in 26 cases by EFTR, with a full-thickness resection rate of $100 \%$ and an average surgery time of $105 \mathrm{~min}$. Dong et al. [15] compared the efficacy of laparoscopic resection vs. EFTR for gastric tumors originating from the muscularis propria and concluded that both approaches 
achieved R0 resection; furthermore, the size of the resected tumors differed insignificantly between them.

It is technically challenging for endoscopists to close a gastric fistula after EFTR. With continuous improvements in endoscopic adjunctive therapeutic equipment, additional methods for gastric defect closure are emerging; OTSC is an example. OTSC is now being used to control gastrointestinal bleeding as well as to close gastrointestinal fistulae and acute gastrointestinal perforation [16-34]. The OTSC system is simple to use and takes minimal time; therefore, it is an appropriate choice for endoscopic wound closure. The literature contains studies of the OTSC, most of which are pilot, experimental, or animal studies; however, to date, the clinical applications of the OTSC have not been fully evaluated. In eight cases, Sarker [3] successfully used the OTSC closure system for the resection of gastrointestinal submucosal tumors (not originating from the muscularis propria) and wound closure. In the study of Fähndrich M, Sandmann M [34], a total of 17 patients underwent EFTR using the OTSC system. The technical success was $94 \%(16 / 17)$. The complete resection (R0) rate was $100 \%$. There were no complications.

In this study, we evaluated the safety and feasibility of the OTSC system for the closure of gastric defects after EFTR for gastric tumors originating from the muscularis propria. Our study results have shown EFTR to be effective for the resection of such tumors. As confirmed by pathology, the full-thickness resection rate of tumors was 23/23 $(100 \%)$, and no residual tumor was observed at the resection margin. This study demonstrated that iatrogenic gastric perforations were successfully closed with OTSC in all 23 cases of EFTR. Furthermore, fistula closure with OTSC significantly shortened the closure time. The average time of gastric defect closure was $4.9 \mathrm{~min}$ in this study.

In many previous studies, metal clips were commonly used to close iatrogenic perforations after gastric resections. Generally speaking, closing small gastrointestinal perforations with metal clips is convenient. Fujishiro et al. [35] reported 27 cases of gastrointestinal perforations at different sites after EMR that underwent closure with metal hemostatic clips. The average diameter of the defects closed was $2.5 \mathrm{~mm}$ (maximum: $5 \mathrm{~mm}$ ), the average closure time was $10 \mathrm{~min}$ (range 8-20 $\mathrm{min}$ ), and the average number of metal clips used was three (range 1-8). However, closing large gastric perforations with metal clips is difficult and time consuming; furthermore, it requires a high level of technical expertise. In addition, there is a certain risk of dehiscence at the closure site following wound closure with metal clips alone; this is because metal clips primarily approximate the gastric mucosa and cannot completely approximate the gastric submucosa and muscularis propria. Considering the above, based on gastric defect closure with metal clips, Ye et al. [4] evaluated the use of nylon suture snares to connect all the metal clips in order to secure their closure. Von Renteln et al. [23] compared the closure effect of conventional endoscopic clips vs. OTSC closure clips on gastric defects and found that the OTSC closure clips were significantly superior to conventional endoscopic clips in terms of the operative time (8.5 vs. $31.5 \mathrm{~min}$ ).

In general, OTSC can rapidly and accurately close gastrointestinal perforations $<20 \mathrm{~mm}$ [2, 16-34]. In a prospective, multicenter study, Voermans et al. [27] treated 36 cases of acute gastrointestinal perforations (including five cases of esophageal perforations, six cases of gastric perforations, 12 cases of duodenal perforations and 13 cases of colon perforations). Of the 36 cases, 33 (89\%) successfully underwent closure with the OTSC closure system and the operative time was $5.44 \pm 4.15 \mathrm{~min}$. In a report by Schlag et al. [28], six patients suffered iatrogenic perforations during endoscopic dissection and resection of gastric tumors; however, wound closure with OTSC was successful in all patients.

OTSC has a rapid, well-established hemostatic effect on gastrointestinal bleeding. Kato et al. [30] prospectively compared the hemostatic effects of three different endoscopic clips on gastrointestinal bleeding and found that OTSC closure clips were superior to conventional metal clips in terms of both the operative time and operative effect; the average operative time of OTSC closure clips was $49.2 \mathrm{~s}$, compared with two brands of conventional metal clips, which required 284.1 and $336.8 \mathrm{~s}$.

The OTSC closure system has a certain restrictive requirement in regard to the perforation size. It is difficult to close a perforation $>3 \mathrm{~cm}[2,31]$, slightly difficult to close a perforation $>2 \mathrm{~cm}$ (complete closure cannot be achieved using one OTSC closure system in some cases) $[2,31]$ and is suitable for closure of a perforation $\leq 2 \mathrm{~cm}$ $[2,16-31]$.

This study has some limitations in regard to the study design; it was a single center, retrospective study based on a small sample size and a short follow-up time. This new technique must be further investigated in larger, multicenter, randomized, controlled, prospective studies.

In conclusion, the OTSC closure system can be used for gastric defect closure after EFTR for gastric tumors originating from the muscularis propria (tumor diameter $\leq 2 \mathrm{~cm}$ ). It is simple to perform, convenient, safe and effective. This new technique requires further investigation.

Acknowledgments This study was supported by the National Natural Science Foundation of China (Grant No. 81071798), Medical Peak Project Foundation of Liaoning Province [Grant No. (2010)696]. We thank all of the doctors, nurses and pathologists who participated in this study. 
Disclosures Jintao Guo, Siyu Sun, Zhijun Liu, Xiang Liu, Sheng Wang, Nan Ge, Guoxin Wang and yafei Qi have no conflicts of interest or financial ties to disclose.

Open Access This article is distributed under the terms of the Creative Commons Attribution License which permits any use, distribution, and reproduction in any medium, provided the original author(s) and the source are credited.

\section{References}

1. Hwang JH, Rulyak SD, Kimmey MB (2006) American Gastroenterological Association Institute technical review on the management of gastric subepithelial masses. Gastroenterology 130:2217-2228

2. Weiland T, Fehlker M, Gottwald T, Schurr MO (2013) Performance of the OTSC System in the endoscopic closure of iatrogenic gastrointestinal perforations: a systematic review. Surg Endosc 27(7):2258-2274

3. Sarker S, Gutierrez JP, Council L, Brazelton JD, Kyanam Kabir Baig KR, Mönkemüller K (2014) Over-the-scope clip-assisted method for resection of full-thickness submucosal lesions of the gastrointestinal tract. Endoscopy 46(9):758-761

4. Ye LP, Yu Z, Mao XL, Zhu LH, Zhou XB (2014) Endoscopic full-thickness resection with defect closure using clips and an endoloop for gastric subepithelial tumors originating from the muscularis propria. Surg Endosc 28(6):1978-1983

5. Guo J, Liu Z, Sun S, Wang S, Ge N, Liu X, Wang G, Liu W (2013) Endosonography-assisted diagnosis and therapy of gastrointestinal submucosal tumors. Endosc Ultrasound 2(3):125-133

6. Mitsunaga, T Tagata, T Hamano, H Teramoto, Y Mitsunaga, I Shirato et al. (2014) The observation indicator induced from gastrointestinal stromal tumor under long-term monitoring by endoscopic ultrasonography. Endosc Ultrasound 3(5): 4-5

7. Kongkam P, Devereaux BM, Ponnudurai R et al (2013) Endoscopic ultrasound forum summary from the Asian Pacific digestive week 2012. Endosc Ultrasound 2:43-60

8. Giovannini M (2012) Report of EUS presentations during the 20th UEGW Meeting in Amsterdam. Endosc Ultrasound $1: 169-172$

9. Liu BR, Song JT, Qu B, Wen JF, Yin JB, Liu W (2012) Endoscopic muscularis dissection for upper gastrointestinal subepithelial tumors originating from the muscularis propria. Surg Endosc 26(11):3141-3148

10. Jeong ID, Jung SW, Bang SJ, Shin JW, Park NH, Kim do H (2011) Endoscopic enucleation for gastric subepithelial tumors originating in the muscularis propria layer. Surg Endosc 25(2): 468-474

11. Shi Q, Chen T, Zhong YS, Zhou PH, Ren Z, Xu MD, Yao LQ (2013) Complete closure of large gastric defects after endoscopic full-thickness resection, using endoloop and metallic clip interrupted suture. Endoscopy 45(5):329-334

12. Zhou PH, Yao LQ, Qin XY, Cai MY, Xu MD, Zhong YS, Chen WF, Zhang YQ, Qin WZ, Hu JW, Liu JZ (2011) Endoscopic fullthickness resection without laparoscopic assistance for gastric submucosal tumors originated from the muscularis propria. Surg Endosc 25(9):2926-2931

13. von Renteln D, Rösch T, Kratt T, Denzer UW, El-Masry M, Schachschal G (2012) Endoscopic full-thickness resection of submucosal gastric tumors. Dig Dis Sci 57(5):1298-1303

14. Wang L, Ren W, Fan CQ, Li YH, Zhang X, Yu J, Zhao GC, Zhao XY (2011) Full-thickness endoscopic resection of nonintracavitary gastric stromal tumors: a novel approach. Surg Endosc 25(2): 641-647
15. Dong HY, Wang YL, Jia XY, Li J, Li GD, Li YQ (2014) Modified laparoscopic intragastric surgery and endoscopic fullthickness resection for gastric stromal tumor originating from the muscularis propria. Surg Endosc 28(5):1447-1453

16. von Renteln D, Rudolph HU, Schmidt A, Vassiliou MC, Caca K (2010) Endoscopic closure of duodenal perforations by using an over-the-scope clip: a randomized, controlled porcine study. Gastrointest Endosc 71:131-138

17. Baron TH, Song LM, Ross A, Tokar JL, Irani S, Kozarek RA (2012) Use of an over-the-scope clipping device: multicenter retrospective results of the first U.S. experience (with videos). Gastrointest Endosc 76(1):202-208

18. Musumba C, Sonson R, Tutticci N, Nanda K, Bourke MJ (2014) Endoscopic submucosal dissection of a duodenal neuroendocrine tumor. Gastrointest Endosc 79(5):716

19. von Renteln D, Denzer UW, Schachschal G, Anders M, Groth S, Rösch T (2010) Endoscopic closure of GI fistulae by using an over-the-scope clip (with videos). Gastrointest Endosc 72(6): 1289-1296

20. Binmoeller KF, Shah JN, Bhat YM, Kane SD (2014) Suck-ligateunroof-biopsy by using a detachable $20-\mathrm{mm}$ loop for the diagnosis and therapy of small subepithelial tumors (with video). Gastrointest Endosc 79(5):750-755

21. Repici A, Arezzo A, De Caro G, Morino M, Pagano N, Rando G, Romeo F, Del Conte G, Danese S, Malesci A (2009) Clinical experience with a new endoscopic over-the-scope clip system for use in the GI tract. Dig Liver Dis 41(6):406-410

22. Chen AI, Lim BS, Ma JS, Chaya CT (2014) Over-the-scope clip for closure of persistent post-esophagectomy gastric conduit fistula. Gastrointest Endosc 79(4):546

23. von Renteln D, Vassiliou MC, Rothstein RI (2009) Randomized controlled trial comparing endoscopic clips and over-the scope clips for closure of natural orifice transluminal endoscopic surgery gastrotomies. Endoscopy 41(12):1056-1061

24. Seebach L, Bauerfeind P, Gubler C (2010) "Sparing the surgeon": clinical experience with over-the-scope clips for gastrointestinal perforation. Endoscopy 42(12):1108-1111

25. Nishiyama N, Mori H, Kobara H, Rafiq K, Fujihara S, Kobayashi M, Oryu M, Masaki T (2013) Efficacy and safety of over-thescope clip: including complications after endoscopic submucosal dissection. World J Gastroenterol 19(18):2752-2760

26. Zhang XL, Sun G, Tang P, Zhang RG, Yang YS (2013) Endoscopic closure of experimental iatrogenic gastric fundus perforation using over-the-scope clips in a surviving canine model. J Gastroenterol Hepatol 28(9):1502-1506

27. Voermans RP, Le Moine O, von Renteln D, Ponchon T, Giovannini M, Bruno M, Weusten B, Seewald S, Costamagna G, Deprez P, Fockens P, CLIPPER Study Group (2012) Efficacy of endoscopic closure of acute perforations of the gastrointestinal tract. Clin Gastroenterol Hepatol 10(6):603-608

28. Schlag C, Wilhelm D, von Delius S, Feussner H, Meining A (2013) EndoResect study: endoscopic full-thickness resection of gastric subepithelial tumors. Endoscopy 45(1):4-11

29. Mennigen R, Colombo-Benkmann M, Senninger N, Laukoetter M (2013) Endoscopic closure of postoperative gastrointestinal leakages and fistulas with the Over-the-Scope Clip (OTSC). J Gastrointest Surg 17(6):1058-1065

30. Kato M, Jung Y, Gromski MA, Chuttani R, Matthes K (2012) Prospective, randomized comparison of 3 different hemoclips for the treatment of acute upper GI hemorrhage in an established experimental setting. Gastrointest Endosc 75(1):3-10

31. von Renteln D, Schmidt A, Vassiliou MC, Rudolph HU, Caca K (2010) Endoscopic full-thickness resection and defect closure in the colon. Gastrointest Endosc 71(7):1267-1273

32. Gorospe EC, Desai S, Al-Bawardy B, Baron TH, Buttar NS, Wong Kee Song LM (2014) Over-the-scope clip closure of 
pancreaticocolonic fistula caused by severe necrotizing pancreatitis. Gastrointest Endosc 79(5):713

33. Irani S, Baron TH, Gluck M, Gan I, Ross AS, Kozarek RA (2014) Preventing migration of fully covered esophageal stents with an over-the-scope clip device (with videos). Gastrointest Endosc 79(5):844-851

34. Fähndrich M, Sandmann M (2014). Endoscopic full-thickness resection for gastrointestinal lesions using the over-the-scope clip system: a case series. Endoscopy. [Epub ahead of print]
35. Fujishiro M, Yahagi N, Kakushima N, Kodashima S, Muraki Y, Ono S, Kobayashi K, Hashimoto T, Yamamichi N, Tateishi A, Shimizu Y, Oka M, Ogura K, Kawabe T, Ichinose M, Omata M (2006) Successful nonsurgical management of perforation complicating endoscopic submucosal dissection of gastrointestinal epithelial neoplasms. Endoscopy 38(10):1001-1006 